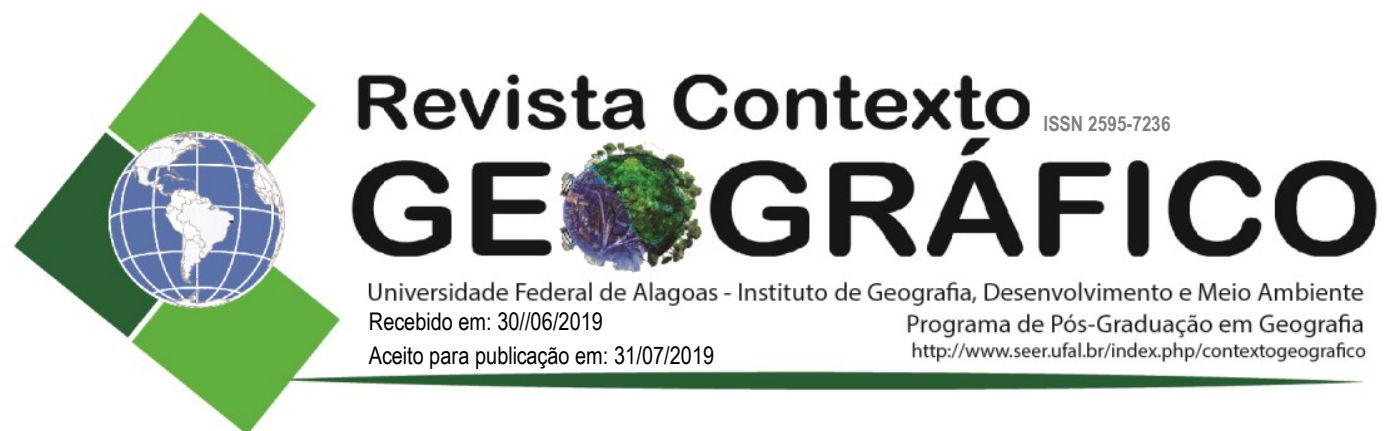

\title{
FUNDAMENTOS TEÓRICOS DE MODELAGEM EM SISTEMAS COMPLEXOS
}

\author{
Mariana Tiné \\ Analista em Geoprocessamento e Cartografia na MRC du Haut-Saint-Laurent, Québec, Canada \\ marianatine@gmail.com
}

Liliana Perez

Professora do Departamento de Geografia, Universidade de Montréal, Canadá

1.perez@umontreal.ca

\author{
Roberto Molowny-Horas \\ Pesquisador Associado do CREAF, Espanha \\ roberto@creaf.uab.cat
}

\begin{abstract}
RESUMO - A geografia tem por definição o estudo do conjunto da Terra, que é por sua natureza extremamente complexo e depende de um número inesgótavel de elementos e relações entre os sistemas que o compõem. A complexidade do mundo atrai cada vez mais a comunidade científica com o inuito de melhor entender e representar as inúmeras interações que ocorrem na superfície, sobretudo no campo da geografia. A partir da teoria da complexidade várias foram as técnicas de modelagem computacional desenvolvidas a fim de simular o mundo real e antecipar possíveis eventos, como por exemplo a expansão urbana de uma determinada cidade, o desmatamento de uma área devido ao avanço da agricultura, e até mesmo padrões de imigração de uma população. É consenso que a modelagem será cada vez mais utilizada no planejamento territorial e ambiental, e este artigo traz uma breve explanação de alguns dos métodos mais comuns utlizados para estes fins.
\end{abstract}

Palavras-chave: sistemas complexos; modelagem; autômatos celulares; modelos baseados no agente; modelos híbridos.

\section{THEORETICAL FOUNDATIONS OF MODELING IN COMPLEX SYSTEMS}

\begin{abstract}
Geography has by definition the study of the Earth as a whole, which is extremely complex in nature and depends on an inexhaustible number of elements and relations between the systems that compose it. The complexity in the world attracts the scientific community in order to better understand and represent the numerous interactions that occur on the surface, especially in geography science. From the complexity theory were several computer modeling techniques developed in order to simulate the real world and anticipate possible events, such as the urban expansion of a city, the deforestation of an area due to the advance of agriculture, and immigration patterns of a population. It is agreed that modeling will be increasingly used in territorial and environmental planning, and this article provides a brief explanation of some of the most common methods used for these purposes.
\end{abstract}

Keywords: complexity systems; modeling; cellular automata; agent-based model; hybrid models.

\section{INTRODUÇÃO}

A geografia como ciência oferece um vasto campo de ação e possibilidades de análise quanto ao estudo dos elementos e das relações que compõem a superfície terrestre (VIDAL DE LA BLACHE, 1913). Essas relações, formadas entre o ser humano e os meios físico e biológico, são os principais fatores de mudanças na superfície da Terra, como também são objeto de estudo da geografia, esses 
elementos formam o espaço geográfico (DANTAS e MEDEIROS, 2008), que inclui uma série de sistemas frágeis, resilientes e complexos.

O espaço geográfico é um todo complexo composto de elementos visíveis, a saber: lugares, redes, espaços, e elementos invisíveis, isto é, as inter-relações entre os lugares e os elementos que compõem sua estrutura. A complexidade do mundo ao nosso redor refere-se aos atores, aos objetos e à multiplicidade de ciclos de realimentação que evoluem sistemas aninhados com fronteiras difusas (MOINE, 2005), sendo o espaço geográfico um lugar onde se pode observar interações naturais ou antrópicas. É possível criar modelos que possam ajudar a melhorar nossa compreensão e descrição do comportamento desses indivíduos, simulando cenários futuros que ajudarão a alcançar uma melhor gestão e compreensão das relações que ocorrem no espaço, por exemplo, os modelos de expansão urbana, que são usados extensivamente para este fim (HAMDY et al., 2016; TAYYEBI et al., 2014).

A complexidade pode ser caracterizada pela presença de um grande número de elementos independentes que interagem, elementos estes que são necessários para reproduzir as funções de sistemas auto-organizados, auto-replicantes, de aprendizagem e adaptativos. Também pode ser descrito como um fenômeno de aparência aleatória induzida por leis simples. É difícil saber a natureza exata dessa complexidade por causa de seu vasto campo de ideias; sua definição estará, portanto, vinculada à perspectiva de cada ciência (MANSON, 2001). Um sistema complexo é uma entidade coerente, de alguma forma reconhecível, mas cujos elementos, interações e dinâmicas geram estruturas e admitem surpresas e novidades que não podem ser definidas a priori (BATTY e TORRENS, 2005). Dito isto, para estudar processos complexos, é possível usar abordagens de modelagem para simular as interações entre indivíduos e seu ambiente e, assim, avaliar os comportamentos resultantes em diferentes escalas, o que permite decidir qual modelo melhor se adapta a cada cenário, visando todas as diversas variáveis (AUMANN, 2007).

Os modelos permitem uma simplificação do mundo real, mas estão longe de ser simples, devido às dificuldades inerentes a cada linha de pensamento e à complexidade inerente dos sistemas. Nos últimos anos, surgiram novas abordagens e técnicas de modelagem (por exemplo, autômatos celulares e modelos baseados em agentes) e seu escopo se expandiu gradualmente à medida que a tecnologia avança. O desenvolvimento de técnicas como inteligência artificial (IA) é cada vez mais utilizada no campo da modelagem ambiental graças ao seu reconhecimento e seu potencial para resolver problemas complexos (CHEN et al., 2008). Os modelos representam, portanto, simplificações nas quais as partes e processos essenciais são simulados. Dada esta definição, existem muitas formas utilizadas para simular e estudar sistemas (ABDOU et al., 2012; AUMANN, 2007; BATTY e TORRENS, 2005). O uso de modelos espaço-temporais facilita o monitoramento, a mudança e a evolução dos fenômenos do meio ambiente. Nos últimos anos, com o desenvolvimento da tecnologia computacional e dos sistemas de informação geográfica (SIG), os pesquisadores que estudam o espaço geográfico e suas dinâmicas estão cada vez mais interessados em usar essa abordagem para determinar padrões de mudança da superfície (ARSANJANI et al., 2015; BATTY, 2012; HYANDYE e MARTZ, 2017; LUO et al., 2015).

Este trabalho não tem como objetivo definir e/ou discutir os conceitos relacionados à teoria da complexidade. O intuito é de apresentar algumas teorias e trabalhos desenvolvidos por todo o mundo, sobretudo na América do Norte e Ásia, que têm como fundamento os métodos e conceitos defendidos pelo geógrafo norte-americano Steven Manson. Estes trabalhos têm auxiliado no estudo e compreensão da dinâmica e relações espaço-temporais na geografia como um todo, como também têm contribuído para as atualizações e discussões na geografia brasileira no que se refere à outras escolas de pensamento geógrafico.

\section{TEORIA DA COMPLEXIDADE}

Estudos da teoria da complexidade aumentaram desde a década de 1990, não há um consenso, porém, quanto à sua definição devido à ampla variedade de ideias relacionadas à complexidade, que varia segundo a perspectiva de cada ciência (MANSON, 2001). Entretanto, é importante 
entender a diferença entre os conceitos de complicado e complexo, pois costumam ser confundidos. Um problema complicado é previsível e linear, ou seja, possui um início, meio e fim bem definidos. Por outro lado, um problema complexo possui um início definido, cujo fim depende das relações e respostas obtidas ao longo do processo. Logo, um sistema complexo é o conjunto de vários sistemas que se relacionam entre si, é uma entidade coerente, de alguma forma reconhecível, cujos elementos, interações e dinâmicas geram estruturas e admitem surpresas e resultados que não podem ser definidos a priori (BATTY e TORRENS, 2005).

Manson (2001), propôs três abordagens para uma melhor compreensão da teoria da complexidade: a complexidade algorítmica, a complexidade determinista e a complexidade agregativa. A primeira abordagem, a complexidade algorítmica (ou computacional) permite explicar matematicamente os problemas. Esta ideia surgiu com a teoria da computação, no início do século $\mathrm{XX}$, quando os matemáticos tentavam desenvolver métodos simples para a resolução de problemas matemáticos utilizando regras padronizadas, ou seja, os modelos computacionais. $\mathrm{O}$ matemático Alan Turing é um dos mais notáveis neste contexto, pois desenvolveu um modelo capaz de realizar operações sequenciais e padronizadas, através de regras e passos determinados, gerando assim, o conceito de algoritmo (LANGLOIS, 2010). Esta abordagem é eficaz ao ser aplicada em problemas práticos, onde é possível determinar o conjunto de instruções, no entanto, existem sistemas que não podem ser explicados com precisão por essa abordagem por terem regras subjetivas, difíceis de se determinar, como por exemplo certas vivências humanas no campo das ciências sociais (MANSON, 2001).

A segunda abordagem, a complexidade determinista está relacionada às Teorias de Catástrofe e do Caos, a primeira refere-se a fenômenos onde o distúrbio mínimo de uma variável no sistema pode gerar caos no resultado, enquanto que a segunda defende que existe uma ordem matemática no caos, que aparentemente é aleatório (MANSON, 2001). É chamada de "determinista", porque os fenômenos podem ser previsíveis e representados por equações matemáticas não-lineares que utilizam o tempo como variável. A modelagem determinista tem como características a sensibilidade às condições iniciais, a noção de feedback, e se baseia nos conceitos matemáticos de bifurcação e de atratores, incluindo os conceitos de sistemas caóticos, onde uma mínima alteração de uma variável pode gerar um resultado desproporcional e imprevisível, como é o caso do "efeito borboleta" definido pelo metereologista Edward Lorentz, onde ele diz que o bater das asas de uma borboleta pode influenciar o sistema climático (FONTES GONÇALVES, 2005). Essa abordagem é amplamente utilizada para simular fenômenos biológicos, climáticos, ambientais e sociais, porém este último com menos intensidade devido à sua complexidade e à limitações de determinar as diversas variáveis.

Por fim, a complexidade agregativa, serve para explicar a sinergia do sistema, onde este é formado a partir da agregação de vários componentes que interagem no tempo e no espaço e onde esse relacionamento irá definir sua complexidade. Os componentes de complexidade agregativa são: a estrutura interna, a qual é definida pela força das interações entre os componentes dos mesmos e com o ambiente externo, a memória, que é a capacidade de registrar as ações dos componentes e permite antecipar reações, o que caracteriza a aprendizagem. Além disso, neste tipo de complexidade o sistema tem a capacidade de se auto-organizar quando há interrupção, permitindolhe sobreviver. Esta abordagem é bastante utilizada no campo da geografia (ALMEIDA e BATTY, 2003; KAMUSOKO e GAMBA, 2015; POOYANDEH e MARCEAU, 2013).

\section{Complexidade e ciência geográfica}

A geografia se ocupa com o estudo de uma estrutura complexa de relações naturais, sociais, políticas, econômicas, entre outras, que são elas próprias espacialmente estruturadas e configuradas ao longo do tempo (O'SULLIVAN, 2004). Segundo Manson e O'Sullivan, (2006), a complexidade é uma questão naturalmente espacial. Os autores usam o termo "estudos baseados-em-espaço-e-lugar" para abranger um grupo mais amplo de ciências, mas que se relacionam em torno dos estudos ambientais e de espaço, como por exemplo a geografia, 
antropologia e a biologia. Baseadas em preocupações compartilhadas, todas estas ciências contribuem para esclarecer o papel da complexidade na compreensão da dinâmica espacial.

De uma forma geral, duas características da complexidade agregativa são frequentemente observadas: auto-organização e memória. Existem muitas interpretações quanto às configurações disciplinares em que esses conceitos foram identificados, mas os principais recursos podem ser descritos de uma perspectiva geográfica (O'SULLIVAN, 2004).

\section{MODELAGEM DE SISTEMAS COMPLEXOS}

O termo "modelo" se tornou bastante comum na aplicação da pesquisa em sistemas complexos. Isto porque através da modelagem é possível entender e estudar diversas variáveis e seus comportamentos através do tempo e do espaço (MANSON e O'SULLIVAN, 2006). Com o avanço da tecnologia das últimas décadas novas abordagens e técnicas de modelagem surgiram, e o seu âmbito tem gradualmente expandido para várias áreas da geografia física, como na avaliação de mudanças na cobertura do solo (AHMED et al., 2013; HATTERMANN et al.; 2008), e na geografia humana, como no estudo de padrões de crescimento urbano (LIU et al., 2015; MUNSHI et al., 2014).

Os modelos, em geral, podem ser espaciais ou não espaciais, estáticos ou dinâmicos, indutivos ou dedutivos, baseados no agente ou baseados no padrão observado, e podem usar uma grande variedade de dados e informações, como imagens classificadas de sensoriamento remoto, variáveis biofísicas e socioeconômicas, indicadores e cenários censitários, etc, que facilmente são incorporados ao SIG e eventualmente utilizados de forma combinada (MAS et al., 2014). Igualmente importante, as técnicas de calibragem e validação dos modelos são fundamentais para que o mesmo seja fiável e útil (RYKIEL, 1996; STRAATMAN et al., 2004; WU, 2002).

Dentre as várias técnicas utilizadas na modelagem de sistemas complexos, podemos destacar os autômatos celulares (ou Cellular Automata - CA) (LANGLOIS, 2008) e modelos baseados no agente (Agent Based Model), que encontrou um interesse real na modelagem dinâmica de questões sociais (BROWN e ROBINSON, 2006). Ainda, baseados em técnicas de inteligência artificial, é possível construir modelos combinando uma ou mais abordagens, os denominados modelos híbridos (ARSANJANI et al., 2012; MUNSHI et al., 2014). A seguir, uma breve descrição destas técnicas de modelagem será apresentada.

\section{Autômatos celulares}

Autômatos celulares (CA) é uma técnica computacional que permite analisar e simular as mudanças espaço-temporais da superfície. Os modelos de autómatos celulares são uma representação do território a ser estudado, contendo células em forma idêntica, cada uma possuindo um estado (ou valor) inicial, que podem corresponder ao habitat, população, etc, que num período de tempo irão mudar simultaneamente de acordo com regras de transição e com os valores das células vizinhas (LANGLOIS, 2010).

Os pioneiros desta técnica foram os matemáticos Alan Turing e John von Neumann. Na década de 1940 Von Neumann criou o conceito de CA e os primeiros computadores, enquanto que Alan Turing desenvolveu uma máquina capaz de decodificar automaticamente mensagens criptografadas durante a Segunda Guerra Mundial. No entanto, a tecnologia disponível na época não conseguia acompanhar o progresso matemático. Foi só em 1970 que o matemático John Conway publicou o conceito CA no jogo da vida, um modelo que tem regras de transição simples para simular a evolução do tempo de uma geração (LANGLOIS, 2008). O uso desse conceito na geografia foi estabelecido apenas na década de 1990 através de estudos de geografia urbana. A partir desse momento, a técnica de CA se tornou popular em várias linhas de pesquisa geográfica (TORRENS e BENENSON, 2005). 
$\mathrm{O}$ modelo de CA inclui quatro elementos: espaço celular, vizinhança, intervalos de tempo e regras de transição (KAMUSOKO e GAMBA, 2015). O espaço é representado por uma grade de células como os pixels de uma imagem, enquanto a vizinhança é um conjunto de células agrupadas de acordo com sua contiguidade. Cada célula tem um estado no momento inicial. Com a progressão do tempo as células mudam de estado simultaneamente (ou restam inalteradas), influenciadas pelo estado das células adjacentes, de acordo com as regras de transição especificadas. Essas regras são um dos elementos mais importantes do modelo, porque definirão os resultados do processo atual.

Autômatos celulares são modelos que consideram o tempo e o espaço como uma montagem de estruturas discretas. Eles têm vários usos, como na simulação de dispersão de incêndios florestais (CÉSAR et al., 2012; GAUDREAU et al., 2016), dinâmica urbana (ARSANJANI et al., 2012; MOGHADAM e HELBICH, 2013; OZTURK, 2015) e mudanças no uso da cobertura da terra (YU et al., 2010; YU et al., 2011). Por serem tão diversos, os CAs podem ser usados em uma ampla variedade de disciplinas.

Utilizando esta abordagem de CA no contexto da ciência geográfica, aplica-se também o conceito de autômato geográfico, que considera regras georreferenciadas para localizar autômatos no espaço. Como característica interna deste tipo de autômato, podemos citar as relações espaciais entre eles e os processos que guiam as mudanças de localização, ou seja, o modelo tem uma relação espacial direta com os processos. Portanto, se a localização dos autômatos mudar, o resultado do modelo também será alterado (TORRENS e BENENSON, 2005).

\section{Modelagem Baseada no Agente}

ABM (Agent Based Model) é um método computacional para criar, analisar e experimentar modelos de agentes interagindo em um ambiente (ABDOU et al., 2012). Os agentes evoluem em um espaço pré-definido ou, ao contrário, estão livres de restrições e interagem entre si. Esta técnica é muito popular entre os pesquisadores que pretendem simular um sistema muito complexo que pode ser modelado globalmente, tendo comportamentos locais conhecidos e simples de formalizar (FILATOVA et al., 2013).

O eixo principal deste método é baseado no conceito de emergência, isto é, o funcionamento do sistema é o resultado do comportamento individual e da interação entre os agentes. Este método é usado em vários campos, como ecologia (BONNELL et al., 2010), ciências sociais (BALBI et al., 2013) e geografia (CROOKS e CASTLE, 2012). Esses modelos exploram como a interação de múltiplos indivíduos pode gerar fenômenos emergentes, mas também como eles podem simular interações mais complexas para melhor entender o sistema (CROOKS e CASTLE, 2012). Esta abordagem é recente em geografia, mas seu potencial tem sido demonstrado e é cada vez mais utilizado na representação das relações entre o agente e o mundo geográfico real, assim como das casas, ruas, etc. (TORRENS e BENENSON, 2005).

\section{Modelos híbridos}

Determinar qual modelo fornece os melhores resultados é difícil porque cada estudo chega a conclusões únicas. Assim, em vez de especificar um único método, a combinação de duas ou mais técnicas deve ser usada, pois pode corrigir as limitações e dar mais poder ao modelo, oferecendo melhores resultados (GHOSH et al., 2017). Por essa razão, estudos baseados no princípio de combinar abordagens em um modelo híbrido e realizar simulações com o método que fornece os melhores resultados estão se tornando cada vez mais populares (OZTURK, 2015). Assim, diversas técnicas já foram associadas a resultados satisfatórios, a saber, os modelos híbridos baseados no agente (CROOKS e CASTLE, 2012; FILATOVA et al., 2013), os modelos de autômatos celulares utilizando a Cadeia Markov (GHOSH et al., 2017; OZTURK, 2015), os conjuntos de Rede 
Neural-Fuzzy (AZARI et al., 2016) e, com a regressão logística (ARSANJANI et al., 2012; HAMDY et al., 2016; LIU et al., 2015; MUNSHI et al., 2014).

Essencialmente, os modelos geoespaciais baseados no agente dependem da localização dos recursos ou fenômenos que estão sendo modelados, de modo que, se um ou mais desses locais mudarem, os resultados mudam completamente (CROOKS e CASTLE, 2012). A parametrização das funções de resposta comportamental nos modelos baseados no agente não são simples, particularmente quando os agentes possuem características complexas e sofisticadas capacidades de tomada de decisão (SMAJGL e BOHENSKY, 2013). Por isso, os $\mathrm{ABM}$ combinados com técnicas georreferenciadas costumam ter mais precisão e produzem resultados mais satisfatórios.

A Cadeia Markov (Markov Chain - MC) é uma técnica em que uma matriz de transição é elaborada a partir da probabilidade de mudança de uma célula num determinado período de tempo, porém ela não leva em consideração a localização espacial das mesmas, sendo comumente empregada com alguma outra técnica complementar, como os autômatos celulares (GRINBLAT et al., 2016). Os modelos CA-MC são bastante eficazes na simulação de mudanças da cobertura e uso do solo, sendo comumente utilizados nestes estudos (GHOSH et al., 2017; KUMAR et al., 2016; TINÉ et al., 2019).

A técnica de Rede Neural-Fuzzy (i.e. difusa) tem sido utilizada na geografia para explicar a inerente incerteza espacial manifestada nos objetos e regiões e como a incerteza pode ser implementada na análise de tais entidades espaciais, como, por exemplo, conduzir o processo de decisão para determinar as células a serem queimadas por um incêndio disseminado ou para controlar as regras de transição para modelar a invasão urbana em terras rurais em um ambiente SIG (BONE et al., 2006). Os estudos que utilizam esta abordagem ilustram a eficácia de usar a teoria Fuzzy para reconhecer incertezas no desenvolvimento de modelos CA e que a definição tradicional discreta de componentes de CA pode ser alterada a fim de superar problemas com conhecimento limitado sobre vários fenômenos ou dados geoespaciais (AZARI et al., 2016).

A regressão logística (RL) é um método usado para calcular a probabilidade de mudança na cobertura da superfície (MUNSHI et al., 2014). Esta abordagem tem sido amplamente utilizada e aceita para a análise de variáveis de resultados binários e em modelos de simulação espacial. A popularidade deste método vem da disponibilidade de softwares fáceis de usar e da facilidade de interpretação dos resultados modelados (ARSANJANI et al., 2012; HOSMER et al., 1997). A RL permite calcular a relação empírica entre as alterações em uma variável dependente binária e várias variáveis preditoras, o que dá a probabilidade de mudança entre classes específicas de cobertura da superfície (TINÉ et al., 2019). Normalmente, as variáveis são derivadas no ambiente SIG e integradas ao modelo de regressão. No entanto, este método não leva em conta a dinâmica espacial (HAMDY et al., 2016). Para resolver esse problema, Wu (2002) propôs pela primeira vez o modelo híbrido de autômatos celulares baseado em regressão logística (CA-RL) para simular mudanças rurais-urbanas na China. Desde então, essa abordagem tem sido utilizada em diversas áreas de estudo, como na expansão urbana (ARSANJANI et al., 2012; LIU et al., 2015; MUNSHI et al., 2014), cobertura e uso do solo (LIN et al., 2011; SOHL et al., 2016). Essa abordagem híbrida ajuda a superar as principais limitações da regressão logística, dando mais poder e credibilidade aos modelos.

\section{CONSIDERAÇÕES FINAIS}

Os sistemas ambientais são estocásticos e, com muita frequência, são processos multiescala, que dependem do espaço e do tempo, eles também tendem a abrigar interações complexas entre processos sociais, culturais, físicos, químicos e biológicos. Esses processos podem não ser bem conhecidos e/ou podem ser difíceis de representar, causando considerável incerteza, algumas das fontes dessa incerteza podem ser superadas com dados adicionais ou investigação adicional, 
mas ela torna-se insuperável, especialmente quando os sistemas são caracterizados por comportamento caótico ou processos auto-organizados.

Com o avanço da tecnologia e uma maior compreensão da complexidade, a representação destes sistemas vem sendo cada vez mais aceita e confiável no que diz respeito à simulação de eventos e comportamentos de um dado problema. A aplicação de técnicas matemáticas e computacionais mostraram ser uma ferramenta importante no campo da geografia, e principalmente no planejamento territorial, pois ela antecipa possíveis situações, proporcionando assim, uma melhor gestão do espaço e de todas as relações inerentes a ele. Entretanto, os modelos devem servir como apoio às tomadas de decisão, pois por mais que sejam fiáveis e bem validados, nunca serão a representação exata dos fenômenos geográficos, sendo o seu resultado sujeito às alterações impossíveis de prever. O modelo será sempre uma simulação e não uma predição dos resultados das diversas interações que ocorrem na complexa superfície terrestre.

\section{AGRADECIMENTOS}

Este artigo é uma versão de um capítulo da dissertação de mestrado defendida por mim na Universidade de Montréal em 2018. Agradeço aos meus orientadores, Dra. Liliana Perez e Dr. Roberto Molowny-Horas por todo ensinamento ao longo do curso. Aos amigos Saeed Harati e Jennifer Sauri meu muito obrigada por todas as trocas e discussões sobre a modelagem de sistemas complexos que foram fundamentais para uma melhor compreensão do meu trabalho. $\mathrm{E}$ finalmente, aos companheiros do Laboratory of Environmental Geosimulation (LEDGE) por todos os debates e aprendizados proporcionados dentro do laboratório e em congressos que participamos em nome do LEDGE

\section{REFERÊNCIAS}

ANDERSON, B.; MCFARLANE, C. Assemblage and geography. Area, v.43, p.124-127, 2011.

ABDOU, M.; HAMILL, L.; GILBERT, N. Designing and Building an Agent-Based Model. In: HEPPENSTALL, A. J. et al. (Eds.). . Agent-Based Models of Geographical Systems. 1. ed. [s.1.] Springer Netherlands, 2012. p. 760.

AHMED, B.; AHMED, R.; ZHU, X. Evaluation of Model Validation Techniques in Land Cover Dynamics. ISPRS International Journal of Geo-Information, v. 2, n. 3, p. 577-597, 2013.

ALMEIDA, C. M. DE; BATTY, M. Stochastic cellular automata modeling of urban land use dynamics: empirical development and estimation. Computers, Environment and Urban Systems, v. 27, p. $481-509,2003$.

ARSANJANI, J. J. et al. Integration of logistic regression, Markov chain and cellular automata models to simulate urban expansion. International Journal of Applied Earth Observation and Geoinformation, v. 21, n. 1, p. 265-275, 2012.

ARSANJANI, T. J. et al. Spatiotemporal monitoring of Bakhtegan Lake's areal fluctuations and an exploration of its future status by applying a cellular automata model. Computers and Geosciences, v. 78, p. 37-43, 2015.

AUMANN, C. A. A methodology for developing simulation models of complex systems. Ecological Modelling, v. 202, n. 3-4, p. 385-396, abr. 2007.

AZARI, M. et al. Integrating cellular automata, artificial neural network, and fuzzy set theory to simulate threatened orchards: application to Maragheh, Iran. GIScience \& Remote Sensing, v. 1603, n. January, p. 1-23, 2016. 
BALBI, S. et al. A spatial agent-based model for assessing strategies of adaptation to climate and tourism demand changes in an alpine tourism destination. Environmental Modelling \& Software, v. 45, p. 29-51, jul. 2013.

BATTY, M. A Generic Framework for Computational Spatial Modelling. In: HEPPENSTALL, A. . (Ed.). . Agent-Based Models of Geographical Systems. [s.1.] Springer Science, 2012. p. 1932.

BATTY, M.; TORRENS, P. M. Modelling and prediction in a complex world. Futures, v. 37, n. 7, p. 745-766, set. 2005.

BONE, C.; DRAGICEVIC, S.; ROBERTS, A. A fuzzy-constrained cellular automata model of forest insect infestations. Ecological Modelling, v. 192, n. 1-2, p. 107-125, fev. 2006.

BONNELL, T. R. et al. An agent-based model of red colobus resources and disease dynamics implicates key resource sites as hot spots of disease transmission. Ecological Modelling, v. 221, n. 20, p. 2491-2500, out. 2010.

BROWN, D.; ROBINSON, D. Effects of heterogeneity in residential preferences on an agentbased model of urban sprawl. Ecology and society, 2006.

CÉSAR, E. S.; VALENTE, M. S.; DIAS, P. V. Modelação Espacial de Incêndios Florestais: autómatos celulares. p. 1-6, 2012.

CHEN, S. H.; JAKEMAN, A. J.; NORTON, J. P. Artificial Intelligence techniques: An introduction to their use for modelling environmental systems. Mathematics and Computers in Simulation, v. 78, n. 2-3, p. 379-400, jul. 2008.

CROOKS, A. T.; CASTLE, C. J. E. The Integration of Agent-Based Modelling and Geographical Information for Geospatial Simulation. In: HEPPENSTALL, A. J. (Ed.). . AgentBased Models of Geographical Systems. [s.1.] Springer Science, 2012. p. 8-10.

DANTAS, A.; MEDEIROS, T. Espaço e Modernidade. In: Introdução à Ciência Geográfica. Natal, Brésil: EDUFRN, 2008. p. 176.

FILATOVA, T. et al. Spatial agent-based models for socio-ecological systems: Challenges and prospects. Environmental Modelling \& Software, v. 45, p. 1-7, jul. 2013.

FONTES GONÇALVES, V. M. COMPLEXIDADE: TEORIA E PRÁTICA INTERDISCIPLINAR. Caderno de Filosofia e Psicologia da Educação, p. 49-75, 2005.

GAUDREAU, J.; PEREZ, L.; DRAPEAU, P. BorealFireSim: A GIS-based Cellular Automata Model of Wildfires for the Boreal Forest of Quebec in a Climate Change Paradigm. Ecological Informatics, v. 32, p. 12-27, 2016.

GHOSH, P. et al. Application of Cellular automata and Markov-chain model in geospatial environmental modeling- A review. Remote Sensing Applications: Society and Environment, v. 5, n. January, p. 64-77, 2017.

GRINBLAT, Y.; GILICHINSKY, M.; BENENSON, I. Cellular Automata Modeling of LandUse/Land-Cover Dynamics: Questioning the Reliability of Data Sources and Classification Methods. Annals of the American Association of Geographers, v. 106, n. 6, p. 1299-1320, 2016.

HAMDY, O. et al. Applying a Hybrid Model of Markov Chain and Logistic Regression to Identify Future Urban Sprawl in Abouelreesh, Aswan: A Case Study. Geosciences, v. 6, n. 4, p. 43, 2016.

HATTERMANN, F. F.; KRYSANOVA, V.; HESSE, C. Modelling wetland processes in regional applications. Hydrological Sciences Journal, v. 53, n. 5, p. 1001-1012, 2008. 
HOSMER, D. W. et al. A COMPARISON OF GOODNESS-OF-FIT TESTS FOR THE LOGISTIC REGRESSION MODEL. STATISTICS IN MEDICINE Ltd. Stat. Med, v. 16, n. 16, p. 965-980, 1997.

HYANDYE, C.; MARTZ, L. W. A Markovian and cellular automata land-use change predictive model of the Usangu Catchment. International Journal of Remote Sensing, v. 38, n. 1, p. 64-81, 2 jan. 2017.

KAMUSOKO, C.; GAMBA, J. Simulating Urban Growth Using a Random Forest-Cellular Automata (RF-CA) Model. ISPRS International Journal of Geo-Information, v. 4, n. 2, p. 447470, 1 abr. 2015.

KUMAR, K. S.; KUMARI, K. P.; BHASKAR, P. U. Application of Markov Chain \& Cellular Automata based model for prediction of Urban transitions. International Conference on Electrical, Electronics, and Optimization Techniques (ICEEOT) - 2016, p. 4007-4012, 2016.

LANGLOIS, P. Cellular automata for modeling spatial systems. The Modeling Process in Geography: From Detreminism to Complexity, p. 278-306, 2008.

LANGLOIS, P. Simulation des systèmes complexes en géographie : fondements théoriques et applications. 1. ed. Paris: Lavoisier, 2010.

LIN, Y.-P. et al. Predictive ability of logistic regression, auto-logistic regression and neural network models in empirical land-use change modeling - a case study. International Journal of Geographical Information Science, v. 25, n. 1, p. 65-87, 2011.

LIU, Y.; DAI, L.; XIONG, H. Simulation of urban expansion patterns by integrating autologistic regression, Markov chain and cellular automata models. Journal of Environmental Planning and Management, v. 58, n. 6, p. 1113-1136, 2015.

LUO, G. et al. Dynamics of landscape patterns in an inland river delta of Central Asia based on a cellular automata-Markov model. Reg Environ Change, v. 15, p. 277-289, 2015.

MANSON, S. Simplifying complexity: a review of complexity theory. Geoforum, v. 32, n. 3, p. 405-414, ago. 2001.

MANSON, S.; O'SUlliVAN, D. Complexity theory in the study of space and place. Environment and Planning A, v. 38, n. 4, p. 677-692, 2006.

MAS, J.-F. et al. Inductive pattern-based land use/cover change models: A comparison of four software packages. Environmental Modelling \& Software, v. 51, p. 94-111, 2014.

MOGHADAM, H. S.; HELBICH, M. Spatiotemporal urbanization processes in the megacity of Mumbai, India: A Markov chains-cellular automata urban growth model. Applied Geography, v. 40, p. 140-149, 2013.

MOINE, A. Le territoire comme un système complexe. Des outils pour 1' aménagement et la géographie. Septièmes rencontres de Théo Quant, janvier 2005, p. 11 P, 2005.

MUNSHI, T. et al. Logistic regression and cellular automata-based modelling of retail, commercial and residential development in the city of Ahmedabad, India. Cities, v. 39, p. 68$86,2014$.

O'SULLIVAN, D. Complexity science and human geography. Transactions of the Institute of British Geographers, v. 29, n. 3, p. 282-295, set. 2004.

OZTURK, D. Urban Growth Simulation of Atakum (Samsun, Turkey) Using Cellular Automata-Markov Chain and Multi-Layer Perceptron-Markov Chain Models. Remote Sensing, v. 7, n. 5, p. 5918-5950, 13 maio 2015.

POOYANDEH, M.; MARCEAU, D. J. A spatial web/agent-based model to support stakeholders' negotiation regarding land development. Journal of environmental management, v. 129C, p. 309-323, 22 ago. 2013. 
RYKIEL, E. J. Testing ecological models: The meaning of validation. Ecological Modelling, v. 90, n. 3, p. 229-244, 1996.

SMAJGL, A.; BOHENSKY, E. Behaviour and space in agent-based modelling: Poverty patterns in East Kalimantan, Indonesia. Environmental Modelling \& Software, v. 45, p. 8-14, 2013.

SOHL, T. et al. Modeled historical land use and land cover for the conterminous United States. Journal of Land Use Science, v. 11, n. 4, p. 476-499, 2016.

STRAATMAN, B.; WHITE, R.; ENGELEN, G. Towards an automatic calibration procedure for constrained cellular automata. Computers, Environment and Urban Systems, v. 28, n. 1-2, p. 149-170, 2004.

TAYYEBI, A.; PERRY, P. C.; TAYYEBI, A. H. Predicting the expansion of an urban boundary using spatial logistic regression and hybrid raster-vector routines with remote sensing and GIS. International Journal of Geographical Information Science, v. 28, n. 4, p. 639-659, 2014.

TINÉ, M.; PEREZ, L.; MOLOWNY-HORAS, R. Hybrid spatiotemporal simulation of future changes in open wetlands: A study of the Abitibi-Témiscamingue region, Québec, Canada. International Journal of Appl Earth Observation and Geoinformation, v. 74, n. July 2018, p. 302-313, 2019.

TORRENS, P. M.; BENENSON, I. Geographic Automata Systems. International Journal of Geographical Information Science, v. 19, n. 4, p. 385-412, abr. 2005.

VIDAL DE LA BLACHE, P. Des caractères distinctifs de la géographie. Annales de Géographie, v. 22, n. 124, p. 289-299, 1913.

WU, F. Calibration of stochastic cellular automata: The application to rural-urban land conversions. International Journal of Geographical Information Science, v. 16, n. 8, p. 795-818, 2002.

YU, H.; HE, Z.; PAN, X. Wetlands shrink simulation using Cellular Automata: A case study in Sanjiang Plain, China. Procedia Environmental Sciences, v. 2, n. 5, p. 225-233, 2010.

YU, J. et al. Cellular automata-based spatial multi-criteria land suitability simulation for irrigated agriculture. International Journal of Geographical Information Science, v. 25, n. 1, p. 131-148, 2011. 